\title{
In-situ TEM observation of bending induced sub-grain boundary formation in copper single crystal
}

Shuang $\mathrm{Li}^{1}$, Lei $\mathrm{Li}^{1}$, Bharat Gwalani ${ }^{2}$, Matthew Olszta ${ }^{3}$, Ayoub Soulami ${ }^{1}$, Peter Sushko ${ }^{4}$, Cynthia Powell $^{2}$, Suveen Mathaudhu ${ }^{5}$, Arun Devaraj ${ }^{4}$ and Chongmin Wang ${ }^{6}$

${ }^{1}$ Pacific Northwest National Laboratory, United States, ${ }^{2}$ Pacific Northwest National Lab, Richland, Washington, United States, ${ }^{3}$ Pacific Northwest National Laboratory, Washington, United States, ${ }^{4}$ PNNL, United States, ${ }^{5}$ University of California - Riverside, United States, ${ }^{6}$ Pacific Northwest National Laboratory, Richland, Washington, United States

Fabrication of metals through severe plastic deformation (SPD)[1] has been noticed to lead to superior properties, of which the origin of the superior properties has been identified to be closely related the shear deformation induced microstructural evolutions. Various techniques based on shear strain have been developed to enhance the properties of metals, such as high pressure torsion, equal channel angular extrusion, twist extrusion, friction stir processing, shear assisted processing and extrusion[2]. Over the past decade, numerous research efforts have been put on exploring the shear deformation induced microstructural evolution of metals through "before" and "after" ex-situ characterizations. For example, deformation of $\mathrm{Cu}$ during surface sliding, with high rate and at cryogenic temperature, is revealed to be dominated by dislocation slip, twining-mediated plasticity, stacking faults, and nanocrystalline layer formation[3-6]. However, there is a lack of understanding of the dynamic shear deformation process and their correlation with crystal defects and thereby microstructural evolution.

Here, we report our direct in-situ TEM observations of bending-induced sub-grain boundary formation processes in $\mathrm{Cu}$ single crystal. The in-situ bending experiment set-up is shown in Figure 1a. The Cu single crystal specimen is prepared by focused ion beam (FIB) lift out. Prior to deformation, the sample is oriented along the [011] zone axis. At the early state of bending, dislocation activity and multiplication appear to be the dominant features. With progression of deformation, stacking faults (SFs) and deformation twins (DTs) nucleate from the site, where the shear strain is maximum for the geometry of specimen used here. With continued bending of the sample, we notice that the DTs grow and propagate towards the interior of the sample. The inward propagation of DT is headed by partial dislocation. It can be noticed that some of the twinning partial dislocations could be arrested, leading to the narrowing of the frontier of the DTs. Cross encountering of propagating DTs with a SF (or nanotwin) that is formed as a consequence of second slip system leads to the formation of boundary inside the single crystal. This boundary, as shown in Figure 1b-e, essentially becomes a sub-grain boundary. From the selected area electron diffraction patterns, the orientation of the two domains that are separated by the sub-grain boundary is confirmed to be around [12-3] and [11-2], respectively. The lattice rotation across the subgrain boundary is about $11^{\circ}$, as shown in the standard stereographic triangle of face-centered cubic crystal (Figure 1f). The direct in-situ TEM observation reveals the key role of DTs in the formation of sub-grain boundary, offering new insights for understanding the microstructure refinement and lattice rotation in $\mathrm{Cu}$ during plastic deformation, especially considering the importance of DTs[7]. 

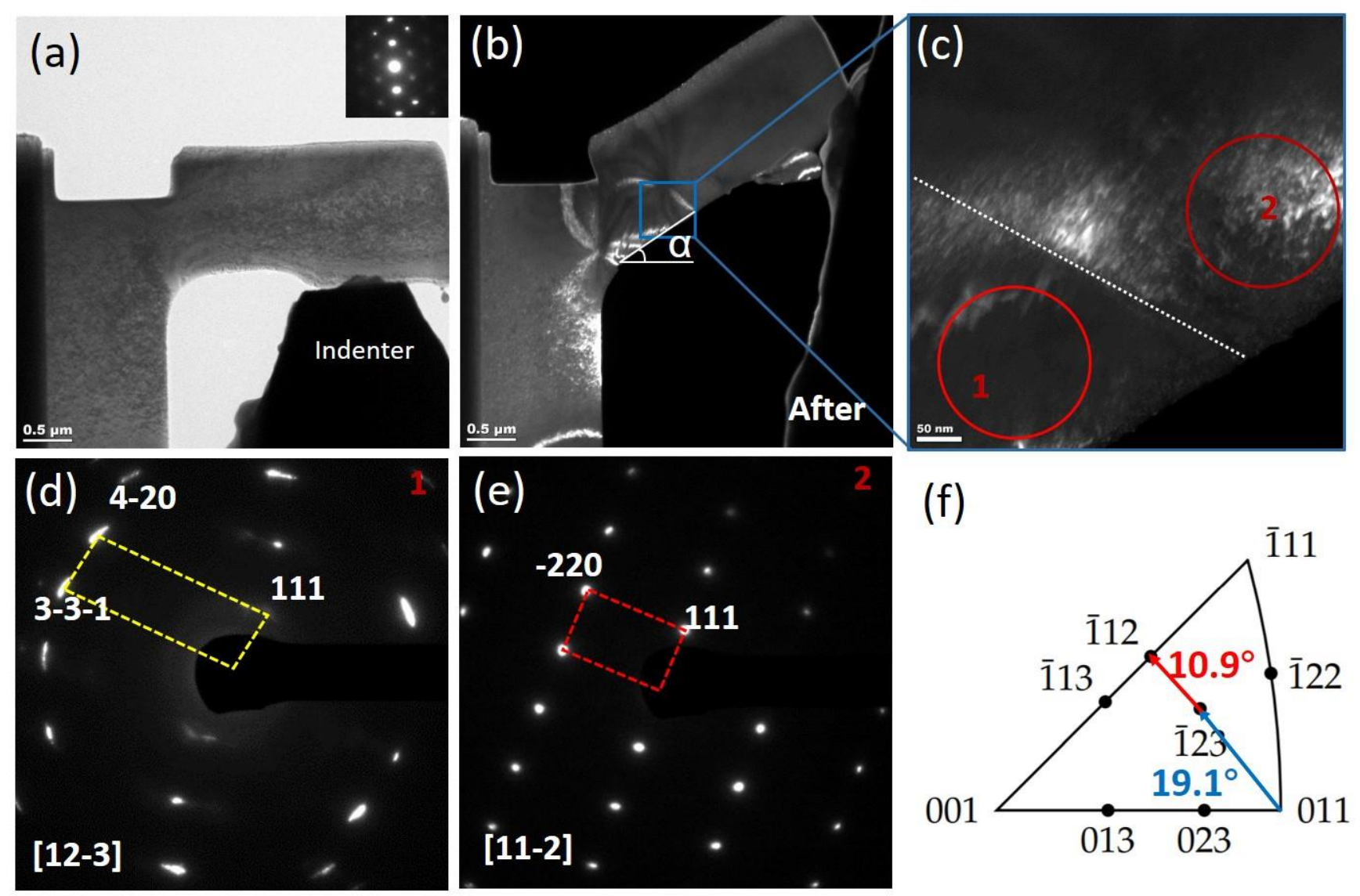

Figure 1. Figure 1. Direct In-situ TEM observation of bending induced sub-grain boundary formation in $\mathrm{Cu}$ single crystal. (a) Bright-field image of in-situ TEM bending set-up with tungsten indenter. The insert is the selected area electron diffraction pattern (SAED), indicating the projection direction of the $\mathrm{Cu}$ single crystal specimen is close to the [110] axis. (b) Dark-field image of the specimen after bending, showing the specimen has rotated about $30^{\circ}$. (c) The enlarged image of the blue rectangle in (b), where the subgrain boundary is marked by the white dash line. (d, e) The SAED of the two regions beside the sub-grain boundary, marked by the red circles in (c). (f) The standard stereographic triangle of face-centered cubic crystal, showing the rotation angle between the different orientation.

\section{References}

[1] C. Wang, et al, Sci. China Technol. Sci., 55 (2012), p. 2377.

[2] G. Faraji, et al, Mater. Sci. Technol., 33 (2017), p.905.

[3] D.A. Hughes, et al, Phys. Rev. Lett., 87 (2001), p.135503.

[4] A. Emge, et al, Wear, 267 (2009), p.562.

[5] X. Chen, et al, Acta Mater., 161 (2018), p.138.

[6] C. Haug, et al, Nat. Commun., 11 (2020), p.839.

[7] The work was conducted in the Environmental Molecular Sciences Laboratory (EMSL), a national scientific user facility sponsored by DOE's Office of Biological and Environmental Research and located at PNNL. 